\title{
Neuronal nitric oxide synthase inhibition and regional sympathetic nerve discharge: implications for peripheral vascular control
}

\author{
Steven W. Copp ${ }^{a}$, Daniel M. Hirai ${ }^{a}$, Gabrielle E. Sims ${ }^{a, b}$, Richard J. Fels ${ }^{a}$, Timothy I. \\ Musch $^{a, b}$, David C. Poole ${ }^{a, b}$, and Michael J. Kenneya \\ aDepartment of Anatomy and Physiology, Kansas State University, Manhattan, KS, 66506, USA \\ bDepartment of Kinesiology, Kansas State University, Manhattan, KS, 66506, USA
}

\begin{abstract}
Neuronal nitric oxide (NO) synthase (nNOS) inhibition with systemically-administered S-methylL-thiocitrulline (SMTC) elevates mean arterial pressure (MAP) and reduces rat hindlimb skeletal muscle and renal blood flow. We tested the hypothesis that those SMTC-induced cardiovascular effects resulted, in part, from increased sympathetic nerve discharge (SND). MAP, HR, and lumbar and renal SND (direct nerve recordings) were measured in 9 baroreceptor (sino-aortic)denervated rats for 20 minutes each following both saline and SMTC $(0.56 \mathrm{mg} / \mathrm{kg}$ i.v.). SMTC increased MAP (peak $\triangle$ MAP: $50 \pm 8 \mathrm{mmHg}, \mathrm{p}<0.01$ ) compared to saline. Lumbar and renal SND were not different between saline and SMTC conditions at any time ( $>>0.05)$. The $\Delta$ SND between saline and SMTC conditions for the lumbar and renal nerves were not different from zero (peak $\Delta$ SND, lumbar: $2.0 \pm 6.8 \%$; renal: $9.7 \pm 9.0 \%$, p $>0.05$ versus zero for both). These data support that SMTC-induced reductions in skeletal muscle and renal blood flow reported previously reflect peripheral nNOS-derived NO vascular control as opposed to increased sympathetic vasoconstriction.
\end{abstract}

\section{Keywords}

sympathetic nervous system; blood pressure; heart rate

\section{Introduction}

Nitric oxide (NO) is an important biological signaling molecule that exerts profound cardiovascular influences. In healthy subjects NO is produced from two distinct constitutively expressed NO synthase (NOS) isoforms: endothelial NOS (eNOS) and neuronal NOS (nNOS). Since the identification of endothelial-derived relaxing factor as NO

\footnotetext{
(C) 2013 Elsevier B.V. All rights reserved.

Corresponding author: Steven W. Copp, Department of Anatomy and Physiology, College of Veterinary Medicine, Kansas State University, Manhattan, KS 66506-5802, Tel.: 785-532-4476, scopp@ vet.ksu.edu.

Publisher's Disclaimer: This is a PDF file of an unedited manuscript that has been accepted for publication. As a service to our customers we are providing this early version of the manuscript. The manuscript will undergo copyediting, typesetting, and review of the resulting proof before it is published in its final citable form. Please note that during the production process errors may be discovered which could affect the content, and all legal disclaimers that apply to the journal pertain.
} 
(reviewed by Moncada and Higgs, 2006), NO-mediated peripheral vascular modulation is often attributed generally to eNOS (Rees et al., 1989; Deanfield et al., 2007). However, studies utilizing mice lacking nNOS (Thomas et al., 1998, 2003; Fadel et al., 2003) and acute pharmacological selective nNOS inhibition (Moore and Handy, 1997; Melikian et al., 2009) have unveiled novel cardiovascular roles specifically for nNOS-derived NO. For example, nNOS-derived NO regulates mean arterial blood pressure (MAP; Narayanan et al., 1995; Komers et al., 2000; Wakefield et al., 2003) via central modulation of sympathetic outflow (Chowdhary and Townend, 1999) and basal (Seddon et al., 2008; Copp et al., 2010) and contracting skeletal muscle (Thomas et al., 1998; Copp et al., 2011) vascular control.

Although a variety of nNOS inhibitors are available (Moore and Handy, 1997), S-methyl-Lthiocitrulline (SMTC) is a particularly potent and selective nNOS inhibitor in vitro and in vivo (Furfine et al., 1994; Narayanan and Griffith, 1994; Wakefield et al., 2003). nNOS inhibition via systemically-administered SMTC in rats evokes global hemodynamic effects including elevations in MAP and reductions in heart rate (HR; Wakefield et al., 2003; Copp et al., 2010, 2011) consequent, at least in part, to peripheral vasoconstriction as evidenced by reductions in hindlimb skeletal muscle and renal blood flow and vascular conductance (VC; Komers et al., 2000; Wakefield et al., 2003; Copp et al., 2010, 2012). It is unknown, however, whether the SMTC-induced hindlimb skeletal muscle and renal vasoconstriction are mediated via alterations in regional sympathetic outflow and increases in lumbar and renal sympathetic nerve discharge (SND), respectively, or, alternatively, reflect peripheral nNOS-derived NO modulation of vascular tone. Moreover, SMTC-induced MAP elevations and concurrent reductions in HR (Wakefield et al., 2003; Copp et al., 2010, 2011) suggest that the baroreflex may mask full expression of the nNOS-derived NO arterial blood pressure and vascular control signals. These are crucial issues to address in order to enhance our understanding of the specific contribution of nNOS-derived NO to cardiovascular control and peripheral vascular regulation.

The purpose of the present investigation was to examine whether nNOS inhibition with systemic SMTC administration impacts regional SND. We tested the specific hypothesis that systemically-administered SMTC in baroreceptor-denervated rats would elevate MAP consequent, at least in part, to increased lumbar and renal SND.

\section{Methods}

\subsection{Animal care and experimental approval}

Nine young adult (4-5 months old, $412 \pm 23 \mathrm{~g}$ ) male Sprague-Dawley rats (Charles River Laboratories, Wilmington, MA) were utilized in the present investigation. Rats were housed 2 per cage in accredited facilities on a 12:12 hour light/dark cycle with standard rat chow and water provided ad libitum. All experimental procedures were approved by the Institutional Animal Care and Use Committee of Kansas State University and conducted according to National Institute of Health guidelines.

\subsection{Surgical preparation}

Anesthesia was induced by isoflurane (3-5\%) and maintained during surgical procedures using isoflurane (1.25-1.75\%), a-chloralose ( $80 \mathrm{mg} / \mathrm{kg}$ i.p.), and urethane (800 mg/kg i.p.). 
Catheters (PE-50) were placed in the femoral vein and femoral artery. Maintenance doses of a-chloralose (35-45 mg/kg/hr) were administered intravenously, whereas maintenance doses of urethane $(200 \mathrm{mg} / \mathrm{kg}$ every $4 \mathrm{~h})$ were administered intraperitoneally. The trachea was cannulated and rats were artificially ventilated. Femoral arterial pressure was monitored using a pressure transducer connected to a blood pressure analyzer. HR was derived from the pulsatile arterial pressure output of the blood pressure analyzer. Core temperature was measured with a rectal thermistor probe and maintained at $\sim 37-38^{\circ} \mathrm{C}$ during surgical interventions by a temperature-controlled table. Adequacy of anesthesia was indicated by an inability of mechanical stimulation of the hindlimb or tail to increase SND or MAP.

To eliminate baroreceptor-mediated afferent feedback that can alter central SND responses, experiments were conducted in baroreceptor (sino-aortic)-denervated (SAD) rats. Bilateral denervation of the aortic arch was completed by cutting the superior laryngeal nerve near its junction with the vagus nerve and removal of the superior cervical ganglion. Bilateral carotid sinus denervation was completed by removal of the adventitia from the area of the carotid sinus bifurcation. SADs were completed 3-4 hours before initiation of experimental protocols. The coherence function relating arterial pressure to SND was used to demonstrate the efficacy of the denervation procedure (Kenney, 1994). Coherence analysis provides a measure of the strength of linear correlation of two signals as a function of frequency. The lack of coherence between arterial pressure and SND at the frequency of the HR demonstrated a complete SAD (Kenney, 1994).

The left renal nerve was isolated retroperitoneally whereas the left lumbar nerve was isolated from a midline approach. Nerve-electrode preparations were covered with silicone gel to prevent exposure to room air. SND was measured and recorded biphasically with a platinum bipolar electrode after capacity-coupled preamplification (bandpass 30-3,000 Hz) from the central end of cut or distally crushed lumbar or renal nerves. Filtered neurograms were routed to an oscilloscope and a nerve traffic analyzer, where sympathetic nerve potentials were full-wave rectified and integrated (10-ms time constant). Total power in lumbar and renal SND was quantified as microvolts $\times$ seconds $(\mu \mathrm{V} \cdot \mathrm{s})$ and SND recordings were corrected for background noise after administration of the ganglionic blocker chlorisondamine ( $5 \mathrm{mg} / \mathrm{kg}$ iv). Technical complications precluded renal SND recordings in 2 rats (final sample size for lumbar SND: $\mathrm{n}=9$, renal SND: $\mathrm{n}=7$ ). A priori power analysis based on previously published data reporting the effects of non-selective NOS inhibition on lumbar and renal SND (Hirai et al., 1995) indicated that a sample size of 6 was required for a statistical power $>0.8$.

\subsection{Experimental protocol}

Anesthetized rats were allowed to stabilize for 60 minutes before initiation of the experimental protocol. Following stabilization, MAP, HR, and lumbar and renal SND were measured and recorded for an $~ 10-15$ minute period where baseline values were determined from the average of the final $\sim 60$ seconds (baseline values are represented as time "zero" in Figure 2 and 3). Rats were then administered, in random order, SMTC $(0.56 \mathrm{mg} / \mathrm{kg}$ dissolved in $0.5 \mathrm{ml}$ heparanized saline; Sigma-Aldrich, St. Louis, MO, USA) or saline (0.5 $\mathrm{ml}$ ) into the femoral vein catheter and MAP, HR and lumbar and renal SND were measured 
and recorded continuously for 20 minutes following each condition. Between experimental conditions baseline values were determined as described above following a second $\sim 5$ minute stabilization period. SMTC is a highly selective inhibitor of nNOS versus eNOS both in vitro and in vivo (Furfine et al., 1994; Narayanan and Griffith, 1994; Wakefield et al., 2003) and this SMTC dose has been utilized recently in our laboratory to assess nNOSmediated cardiovascular and skeletal muscle function in healthy (Copp et al., 2010, 2011), heart failure (Copp et al., 2012), and senescent (Hirai et al., 2012) rats (see Experimental considerations for further details regarding efficacy and selectivity of nNOS inhibition with SMTC). In one rat, a short bout of combined hypoxia and hypercapnia (i.e., shutting off the mechanical ventilator for $\sim 20$ seconds) was implemented as a positive control following saline and SMTC in which it was confirmed that a physiological increase in SND could be detected following completion of the experimental protocol (Figure 1 inset). There were no qualitative or quantitative differences in MAP, HR, or lumbar and renal SND depending on the order of experimental conditions. For each rat the entire experiment (including surgery, stabilization, and experimental protocol) lasted $\sim 6-8$ hours. Following the experimental protocol rats were euthanized by an overdose of methohexital sodium (150 mg/kg i.v.).

\subsection{Statistical analysis}

Data are expressed as mean \pm SEM. MAP, HR, and lumbar and renal SND were compared between saline and SMTC conditions via paired Student's t-test (uncorrected for multiple comparisons). The differences between saline and SMTC conditions (i.e., SMTC-saline) were compared to "zero" via z-tests (Colton 1974). This multi-statistical approach was utilized in order to improve the ability to detect a difference between saline and SMTC conditions should one exist (see Experimental considerations for more details). Significance was accepted at $\mathrm{p}<0.05$.

\section{Results}

\subsection{Effects of nNOS inhibition with SMTC on lumbar and renal SND}

Original tracings of lumbar and renal SND recordings for the different experimental conditions from a representative rat are shown in Figure 1. Note the similarity among baseline, saline, and SMTC conditions whereas subsequent combined hypoxia and hypercapnia markedly augmented SND (Figure 1 inset) which serves as a positive physiological control and establishes that increased SND, should it occur, can be detected. Specifically, there were no differences between saline and SMTC conditions for lumbar or renal SND at any time (Figure 2, top panels). The $\triangle$ SND between saline and SMTC conditions (SMTC-saline) for the lumbar and renal nerve were not different from zero at any time (Figure 2, bottom panels).

\subsection{Effects of nNOS inhibition with SMTC on MAP and HR}

SMTC administration increased MAP significantly compared to saline (Figure 3). Specifically, significant MAP elevations compared to saline were evident within 1 minute following SMTC infusion and this significant elevation persisted for 10 minutes. MAP was not different between saline and SMTC conditions at 15 and 20 minutes following infusion. HR was not different between saline and SMTC conditions at any time (Figure 3). 


\section{Discussion}

The principal novel finding of the present investigation is that nNOS inhibition with systemic SMTC administration evoked marked increases in MAP but, contrary to our hypothesis, did not impact lumbar or renal SND in SAD rats. This finding suggests an important role for peripheral nNOS-derived NO in the regulation of MAP and vascular control. Specifically, these data support that systemic SMTC-induced reductions in rat hindlimb skeletal muscle and renal blood flow and VC observed previously by our laboratory (Copp et al., 2010, 2012) and others (Komers et al., 2000; Wakefield et al., 2003) reflected substantial peripheral nNOS-derived NO vascular control in those tissues. The present investigation contributes significantly to our understanding of cardiovascular regulation via peripheral nNOS-derived NO and has broad implications for chronic disease conditions associated with altered nNOS localization and/or function (Villanueva and Giulivi, 2010).

\subsection{Effects of SMTC on MAP}

Systemic SMTC administration in SAD rats induced increases in MAP $(\uparrow \sim 41 \%)$ which were markedly greater than reported previously in conscious and anesthetized baroreceptor-intact rats from our laboratory ( $\uparrow \sim 10-12 \%$, Copp et al., 2010, 2011) and others (Narayanan et al., 1995; Komers et al., 2000; Wakefield et al., 2003). The significant reductions in HR in those previous reports (Komers et al., 2000; Wakefield et al., 2003; Copp et al., 2010, 2011) and the greater MAP increases in SAD rats herein reveal that peripheral nNOS-derived NO pressure regulation is partially masked following systemic SMTC administration in baroreceptor-intact rats. In addition, the lack of any increase in lumbar and renal SND identify that SMTC-induced peripherally-mediated vasoconstriction underlies a substantial portion of the MAP increase.

The rapid (i.e., within 1 minute) SMTC-induced MAP elevations reported herein peaked after 2 minutes and then returned to values not different from saline within 15 minutes. This is consistent with transient SMTC-induced MAP elevations reported previously (Komers et al., 2000; Wakefield et al., 2003). Importantly, in recent investigations from our laboratory MAP, HR, and skeletal muscle and kidney blood flow were measured $~ 4-5$ minutes following SMTC administration (Copp et al., 2010, 2011, 2012; Hirai et al., 2012). Thus, the present data support that those measurements occurred when the cardiovascular effects of SMTC were peaking.

\subsection{Effects of systemic SMTC on lumbar and renal SND}

Systemic SMTC administration in SAD rats did not alter lumbar or renal SND compared to saline. This suggests that systemically-administered SMTC-induced reductions in hindlimb skeletal muscle ( $\downarrow \sim 33 \%$; Copp et al., 2010) and renal ( $\downarrow \sim 37-40 \%$; Copp et al., 2010, 2012) blood flow and VC reflect important peripheral nNOS-derived NO vascular control. Furthermore, given the use of rats with intact-baroreceptors in those previous investigations, the reductions in blood flow and $\mathrm{VC}$ were, if anything, an underestimation of the peripheral nNOS-derived NO vascular control signal. 
The present data should not be interpreted to reflect the lack of a role for nNOS-derived NO in the regulation of sympathetic outflow. It has been demonstrated clearly that direct intracerebroventricular SMTC administration elevates MAP and enhances renal SND (Tandai-Hiruma et al., 2005) which is consistent with an overall sympathoinhibitory role of central nNOS-derived NO (Chowdhary and Townend, 1999; Patel et al., 2001). The present protocol cannot specifically delineate central versus peripheral effects of SMTC on MAP. However, in conjunction with previous reports (Wakefield et al., 2003; Copp et al., 2010), the lack of any differences between lumbar and renal SND in the present investigation support that peripheral nNOS derived-NO constitutes an important controller of hindlimb skeletal muscle and renal vascular tone at rest and, therefore, serves as a critical modulator of basal blood pressure. Interestingly, the obligatory peripheral nNOS-derived NO vascular signal is lost in the active hindlimb skeletal muscle vasculature during low-speed treadmill running (Copp et al., 2010) where eNOS-derived NO presumably becomes the predominant NO signal in response to endothelial shear stress. In contrast, obligatory peripheral nNOSderived NO vascular control is preserved in the renal vasculature during treadmill running (Copp et al., 2010) and in the anesthetized rat spinotrapezius muscle during electricallyinduced contractions (Copp et al., 2011).

\subsection{Experimental considerations}

A crucial consideration of the current experimental approach is the efficacy of achieving nNOS inhibition without impacting eNOS-mediated function. Several key lines of evidence support this was achieved in the present investigation. First, SMTC is a potent nNOS inhibitor which possesses a 17 -fold selectivity for nNOS over eNOS in rat tissue in vivo (Furfine et al., 1994) and induced significant increases in MAP herein. Second, $0.56 \mathrm{mg} / \mathrm{kg}$ of SMTC (the identical dose utilized herein) does not attenuate the hypotensive response to rapid acetylcholine (ACh) infusion whereas it is blunted (smaller $\triangle \mathrm{MAP}$ and faster recovery time) following non-selective NOS inhibition with L-NAME (Copp et al., 2010, 2011, 2012; Hirai et al., 2012). This is a crucial observation given that vasodilation in response to $\mathrm{ACh}$ is due, in large part, to eNOS-derived NO. Had the SMTC dose in those reports been so high that eNOS was inhibited it would have manifested as an attenuated hypotensive response similar to that seen following L-NAME. These important points suggest efficacious nNOS inhibition occurred in the absence of any effects on eNOS-mediated function.

The less conservative statistical approach using t-tests uncorrected for multiple comparisons and z-tests increased our ability to detect a difference between saline and SMTC conditions should one have existed. However, by these analyses there was no effect of SMTC on SND. Applying the more conservative analyses via t-tests with Bonferroni correction and ANOVAs did not change this conclusion. Post hoc analyses revealed that 477 rats would be needed to detect statistically-significant SMTC-induced increases in lumbar and renal SND.

Even if this number of experiments were to be performed, the extremely small differences in SND would not be expected to contribute physiologically to the marked SMTC-induced reductions in hindlimb skeletal muscle ( $\downarrow \sim 33 \%$; Copp et al. 2010) or renal ( $\downarrow \sim 37-40 \%$; Copp et al., 2010, 2012) blood flow and VC reported previously. 


\subsection{Perspectives and conclusions}

Selective nNOS inhibition with systemically-administered SMTC markedly elevated MAP without altering lumbar or renal SND. Previous investigations have demonstrated that SMTC-induced MAP elevations occur simultaneous with reductions in rat hindlimb skeletal muscle and renal blood flow and VC (Wakefield et al., 2003; Copp et al., 2010, 2012). Therefore, the present data reveal that those MAP elevations and peripheral vasoconstriction were likely not secondary to enhanced lumbar and renal SND but, rather, that they reflect important peripheral nNOS-derived NO vascular control. This contributes significantly to our current understanding of nNOS-derived NO cardiovascular modulation and challenges the conventional notion that eNOS-derived NO constitutes the principal basal NO cardiovascular control signal. This concept/regulation has important implications for chronic disease conditions associated with altered nNOS localization/function and/or impaired vascular control (Villanueva and Giulivi, 2010).

\section{Acknowledgments}

The authors thank Ms. Melanie A. Ray for technical assistance. SWC is supported by an American Heart Association (AHA) Midwest Affiliate Pre-doctoral Fellowship. This work was supported by NIH grant AG-041948 to MJK and NIH grant HL-108328 and AHA Midwest Affiliate grant 10GRNT4350011 to DCP.

\section{References}

Chowdhary S, Townend JN. Role of nitric oxide in the regulation of cardiovascular autonomic control. Clin Sci. 1999; 97:5-17. [PubMed: 10369789]

Colton, T. Statistics in Medicine. Little, Brown and Company; Boston, MA: 1974.

Copp SW, Hirai DM, Ferguson SK, Holdsworth CT, Musch TI, Poole DC. Effects of chronic heart failure on neuronal nitric oxide synthase-mediated control of microvascular $\mathrm{O}_{2}$ pressure in contracting rat skeletal muscle. J Physiol. 2012; 590:3585-3596. [PubMed: 22687613]

Copp SW, Hirai DM, Ferguson SK, Musch TI, Poole DC. Role of neuronal nitric oxide synthase in modulating microvascular and contractile function in rat skeletal muscle. Microcirculation. 2011; 18:501-511. [PubMed: 21535296]

Copp SW, Hirai DM, Schwagerl PJ, Musch TI, Poole DC. Effects of neuronal nitric oxide synthase inhibition on resting and exercising hindlimb muscle blood flow in the rat. J Physiol. 2010; 588:1321-1331. [PubMed: 20176629]

Deanfield JE, Halcox JP, Rabelink TJ. Endothelial function and dysfunction: testing and clinical relevance. Circulation. 2007; 115:1285-1295. [PubMed: 17353456]

Fadel PJ, Zhao W, Thomas GD. Impaired vasomodulation is associated with reduced neuronal nitric oxide synthase in skeletal muscle of ovariectomized rats. J Physiol. 2003; 549:243-253. [PubMed: 12665606]

Furfine ES, Harmon MF, Paith JE, Knowles RG, Salter M, Kiff RJ, Duffy C, Hazelwood R, Oplinger JA, Garvey EP. Potent and selective inhibition of human nitric oxide synthases. Selective inhibition of neuronal nitric oxide synthase by S-methyl-L-thiocitrulline and S-ethyl-L-thiocitrulline. J Biol Chem. 1994; 269:26677-26683. [PubMed: 7523410]

Hirai DM, Copp SW, Holdsworth CT, Ferguson SK, Musch TI, Poole DC. Effects of neuronal nitric oxide synthase inhibition on microvascular and contractile function in skeletal muscle of aged rats. Am J Physiol Heart Circ Physiol. 2012; 303:H1076-1084. [PubMed: 22923618]

Hirai T, Musch TI, Morgan DA, Kregal KC, Claassen DE, Pickar JG, Lewis SJ, Kenney MJ. Differential sympathetic nerve responses to nitric oxide synthase inhibition in anesthetized rats. Am J Physiol Regul Integr Comp Physiol. 1995; 269:R807-R813.

Kenney MJ. Frequency characteristics of sympathetic nerve discharge in anesthetized rats. Am J Physiol. 1994; 267:R830-840. [PubMed: 8092329] 
Komers R, Oyama TT, Chapman JG, Allison KM, Anderson S. Effects of systemic inhibition of neuronal nitric oxide synthase in diabetic rats. Hypertension. 2000; 35:655-661. [PubMed: 10679513]

Melikian N, Seddon MD, Casadei B, Chowienczyk PJ, Shah AM. Neuronal nitric oxide synthase and human vascular regulation. Trends Cardiovasc Med. 2009; 19:256-262. [PubMed: 20447567]

Moncada S, Higgs EA. The discovery of nitric oxide and its role in vascular biology. Br J Pharmacol. 2006; 147(Suppl 1):S193-201. [PubMed: 16402104]

Moore PK, Handy RL. Selective inhibitors of neuronal nitric oxide synthase--is no NOS really good NOS for the nervous system? Trends Pharmacological Sci. 1997; 18:204-211.

Narayanan K, Griffith OW. Synthesis of L-thiocitrulline, L-homothiocitrulline, and S-methyl-Lthiocitrulline: a new class of potent nitric oxide synthase inhibitors. J Medicinal Chem. 1994; 37:885-887.

Narayanan K, Spack L, McMillan K, Kilbourn RG, Hayward MA, Masters BS, Griffith OW. S-alkylL-thiocitrullines. Potent stereoselective inhibitors of nitric oxide synthase with strong pressor activity in vivo. J Biol Chem. 1995; 270:11103-11110. [PubMed: 7538112]

Patel KP, Li YF, Hirooka Y. Role of nitric oxide in central sympathetic outflow. Exp Biol Med. 2001; 226:814-824.

Rees DD, Palmer RM, Moncada S. Role of endothelium-derived nitric oxide in the regulation of blood pressure. Proc Natl Acad Sci. 1989; 86:3375-3378. [PubMed: 2497467]

Seddon MD, Chowienczyk PJ, Brett SE, Casadei B, Shah AM. Neuronal nitric oxide synthase regulates basal microvascular tone in humans in vivo. Circulation. 2008; 117:1991-1996. [PubMed: 18391107]

Tandai-Hiruma M, Horiuchi J, Sakamoto H, Kemuriyama T, Hirakawa H, Nishida Y. Brain neuronal nitric oxide synthase neuron-mediated sympathoinhibition is enhanced in hypertensive Dahl rats. $\mathrm{J}$ Hypertens. 2005; 23:825-834. [PubMed: 15775788]

Thomas GD, Sander M, Lau KS, Huang PL, Stull JT, Victor RG. Impaired metabolic modulation of alpha-adrenergic vasoconstriction in dystrophin-deficient skeletal muscle. Proc Natl Acad Sci. 1998; 95:15090-15095. [PubMed: 9844020]

Thomas GD, Shaul PW, Yuhanna IS, Froehner SC, Adams ME. Vasomodulation by skeletal musclederived nitric oxide requires alpha-syntrophin-mediated sarcolemmal localization of neuronal Nitric oxide synthase. Circ Res. 2003; 92:554-560. [PubMed: 12600881]

Villanueva C, Giulivi C. Subcellular and cellular locations of nitric oxide synthase isoforms as determinants of health and disease. Free Radic Biol Med. 2010; 49:307-316. [PubMed: 20388537]

Wakefield ID, March JE, Kemp PA, Valentin JP, Bennett T, Gardiner SM. Comparative regional haemodynamic effects of the nitric oxide synthase inhibitors, S-methyl-L-thiocitrulline and LNAME, in conscious rats. Br J Pharmacol. 2003; 139:1235-1243. [PubMed: 12871844] 


\section{Highlights}

- Previous reports show that nNOS inhibition (SMTC) reduces skeletal muscle and renal blood flow

- This may reflect central ( $\uparrow$ sympathetic nerve discharge, SND) or peripheral nNOS-mediated effects

- Presently, systemically-administered SMTC elevated MAP without impacting lumbar or renal SND

- This, in conjunction with previous reports, supports important peripheral nNOSderived NO vascular control 


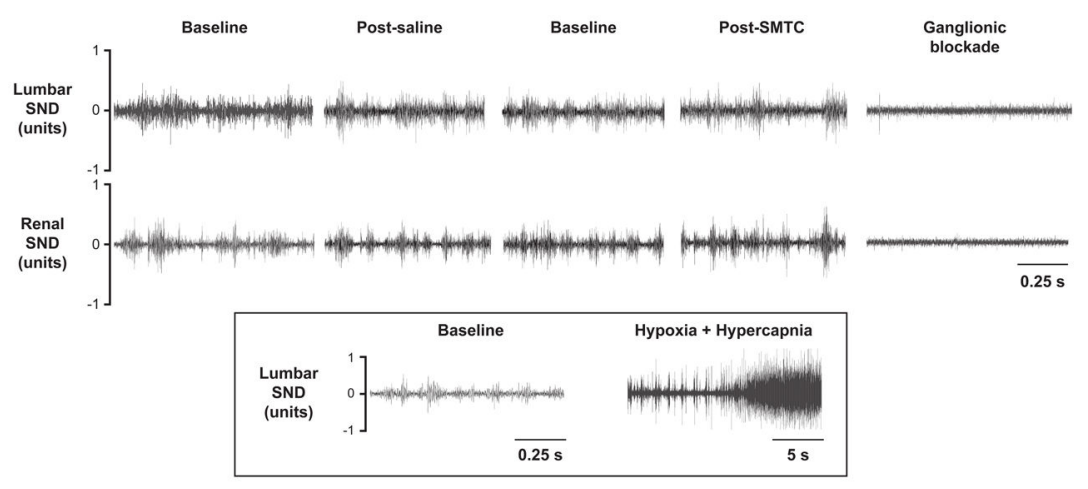

Figure 1.

Original tracings of lumbar and renal sympathetic nerve discharge (SND) recordings for the baseline periods, saline and SMTC $(0.56 \mathrm{mg} / \mathrm{kg})$ conditions, as well as following ganglionic blockade via chlorisondamine from a representative rat. The inset shows an original lumbar nerve recording in which combined hypoxia and hypercapnia were implemented (following saline and SMTC) to provide a positive physiological control and establish that increased SND, should it occur, can be detected. 

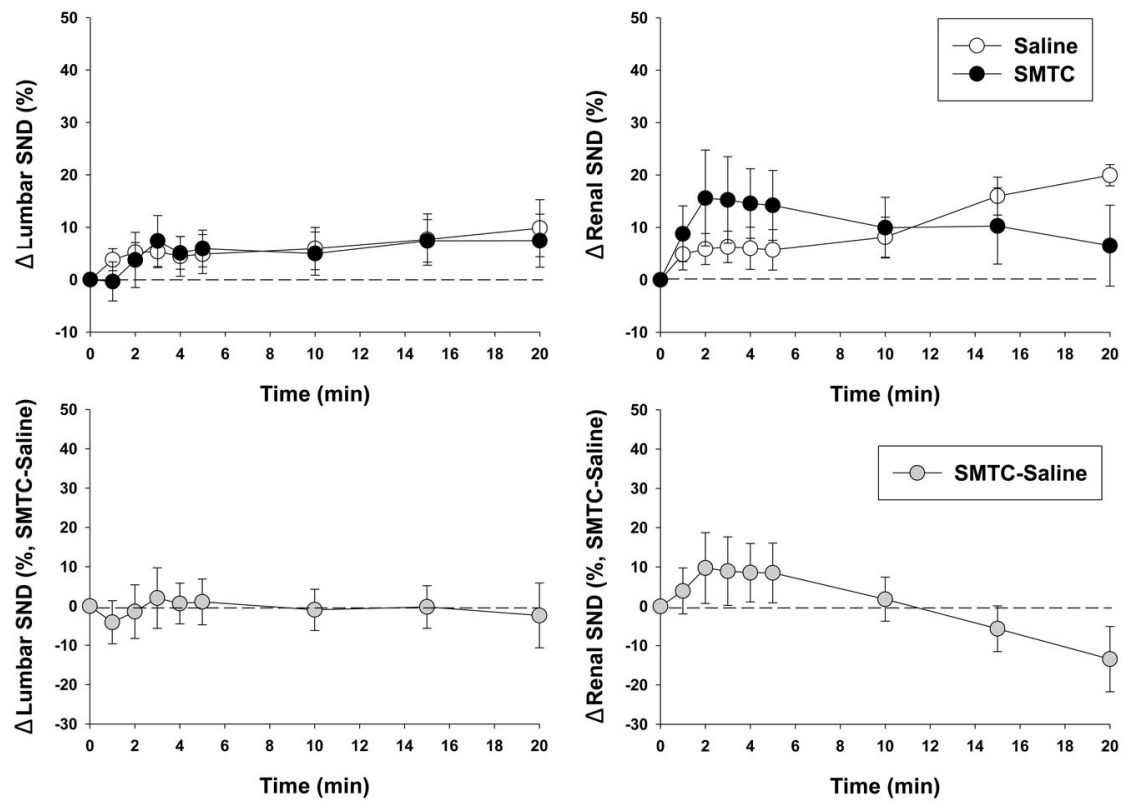

Figure 2.

Effects of saline and SMTC $(0.56 \mathrm{mg} / \mathrm{kg})$ infusions on lumbar $(\mathrm{n}=9)$ and renal $(\mathrm{n}=7)$ SND. There were no differences between saline and SMTC at any time. Time "zero" represents pre-saline or SMTC infusion baseline values. Bottom panels depict the differences between saline and SMTC conditions ( $\triangle$ SND, SMTC-saline). There were no differences from zero at any time. Data are expressed as mean \pm SEM. 

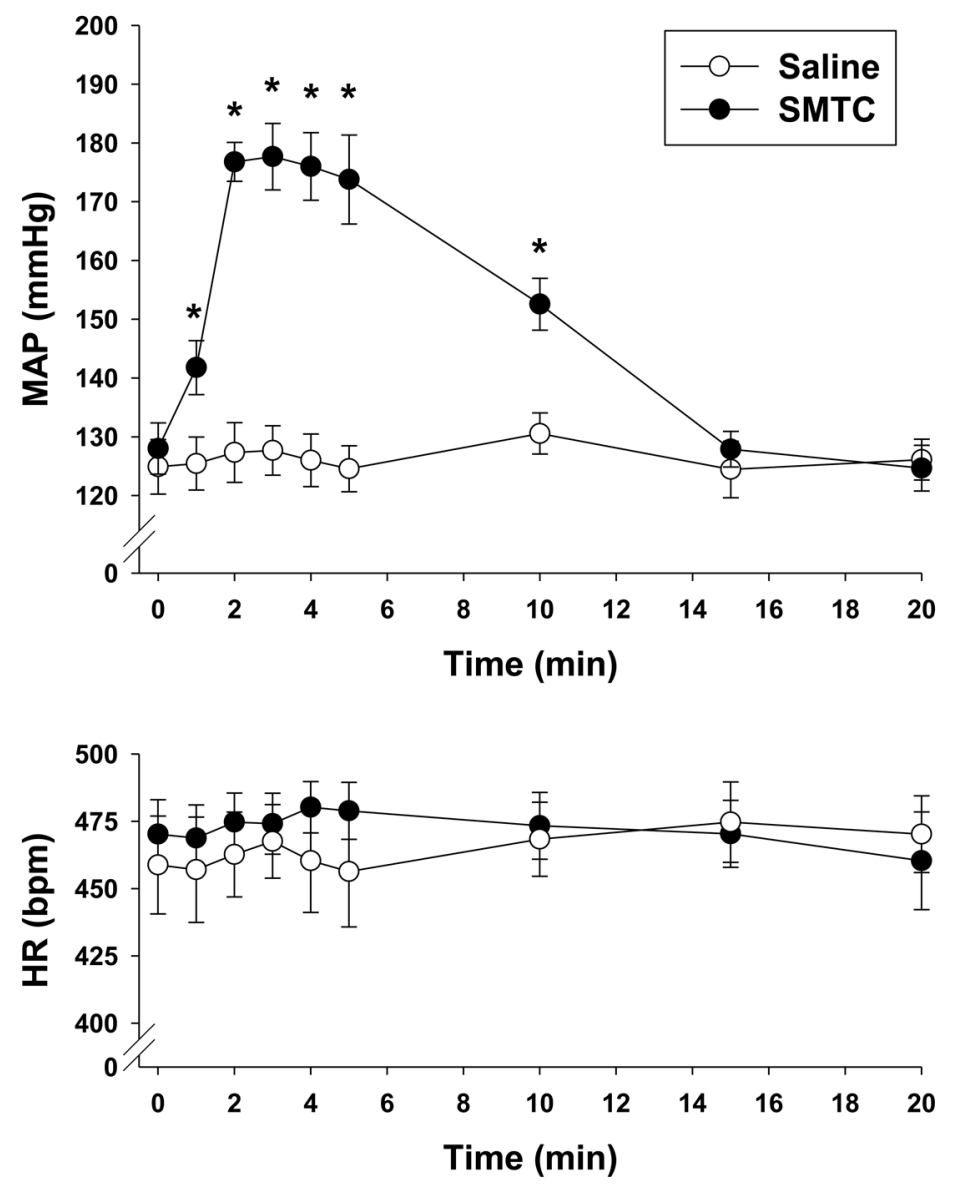

Figure 3.

Effects of saline and SMTC $(0.56 \mathrm{mg} / \mathrm{kg})$ infusions on MAP and HR (n=9). Time "zero" represents pre-saline or SMTC infusion baseline values. Data are expressed as mean \pm SEM. * $\mathrm{p}<0.05$ versus saline. 